\title{
RELATIONSHIP BETWEEN SERUM LEVEL OF IONIZED CALCIUM, MAGNESIUM, PHOSPHATE, VITAMIN D AND PARATHYROID HORMONE WITH STAGES OF CKD.
}

\author{
${ }^{1}$ M ohammed A, ${ }^{2}$ Suleiman H M, , ${ }^{2}$ Yusuf $R,{ }^{2}$ Aliyu IS, ${ }^{2}$ Ibrahim A, ${ }^{3}$ Jibril ME, ${ }^{2}$ M anu
}

${ }^{1}$ Department of Chemical Pathology FMC Bida

${ }^{2}$ Departments of Chemical PathologyA hmadu Bello University Zaria

${ }^{3}$ Department of MedicineA hmadu Bello University Zaria

\author{
Correspondences and reprint request to A uthor: Dr H afsatu M. Suleiman, Department of \\ Chemical Pathology, A hmadu Bello University Zaria, PMB 1044 Samaru Zaria, \\ Kaduna State, Nigeria.
}

E-mail: suleimanhafsatu@yahoo.com, suleimanhafsatu79@gmail.com Tel: +2348037013611

\begin{abstract}
Background: Chronic kidney disease is defined as either damage or a decreased Glomerular Filtration Rate of less than $60 \mathrm{ml} / \mathrm{min} / 1.73 \mathrm{~m}^{2}$ for 3 or more months. There is destruction of renal mass with irreversible sclerosis and loss of nephron leading to a progressive decline in GFR.Secondary hyperparathyroidism hyperphosphataemia, hypocalcaemia and vitamin-D deficiency are common complications of CKD. O bjective: To determine relationship between serum level of ionised calcium, magnesium, phosphate, vitamin-D and parathyroid hormone with stages of CKD. M ethod: This study was conducted at ABUTH Zaria. 125 consecutiveadult patients in various stages of CKD who presented were enrolled and 125 apparently healthy matched for sex and age controls wereal so recruited. R esults: $9 \%$ of patients werein stage $1,16 \%$ in stage $2,22 \%$ in stage $-3,12 \%$ in stage -4 and $41 \%$ in stage 5 . Serum ionised calcium, vitamin-D and $\mathrm{eCrCl}$ showed a progressive dedine as the stage of CKD advances, while serum phosphate, creatinine and iPTH showed a progressive increase as the stage of CKD advances. Changes in serum magnesium showed a slight changew ith advancing stages of CKD. The differencein mean serum levels of calcium, phosphorus, vitamin-D, parathyroid hormone, creatinine and eCrCl with different stages of CKD were statistically significant. $\mathrm{eCrCl}$ correlated negatively with phosphate and iPTH while serum creatinine correlated negatively with calcium and positively with phosphate and iPTH. Conclusion: Majority of CKD patients were in late stage. Correlation of analytes with stages was more in late stages and biochemical derangements occurred in late, rather than early stages of CKD.
\end{abstract}

Keyw ords: Chronickidney disease, Calcium, Phosphate, Vitamin D, Magnesium, Parathyroid hormone

\section{INTRODUCTION}

Chronic damage to the kidney also known as chronic kidney disease (CKD) has serious implication for systematic functions, growth and existence. ${ }^{1}$ Chronic kidney disease(CKD) is defined

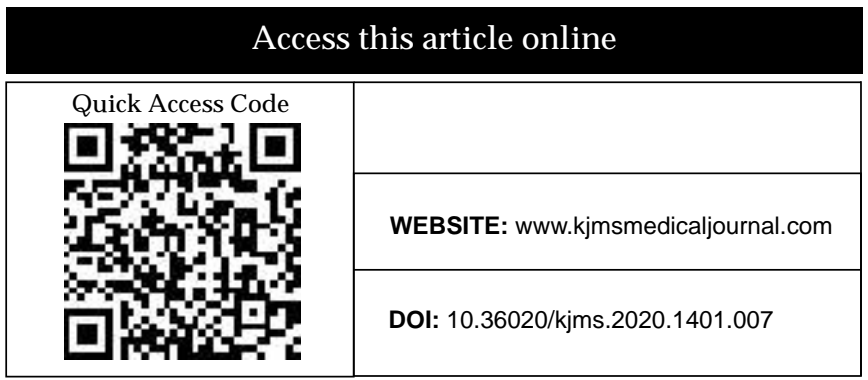

as either damage or a decreased Glomerular Filtration Rate (GFR) of less than $60 \mathrm{ml} / \mathrm{min} / 1.73 \mathrm{~m}^{2}$ for 3 or more months. With underlying aetiology, the destruction of renal mass with irreversible sclerosis and loss of nephron leads to a progressive decline in GFR. The different stages of kidney diseaseforma continuumin time. ${ }^{2}$

In $2002 \mathrm{~K} / \mathrm{DOQ}$ published its first classification of the stages of chronic kidney disease (which was updated in 2012) asfollows.

- Stage1; kidney damagewith normal or increased GFR $(\geq 90 \mathrm{ml} / \mathrm{min}$ 


\section{( $\left.1.73 m^{2}\right)$}

- $\quad$ Stage 2; kidney damage with mild reduction in GFR (60$89 \mathrm{ml} / \mathrm{min} / 1.73 \mathrm{~m}^{2}$ )

- $\quad$ Stage 3; moderate reduction in GFR $\left(30-59 \mathrm{ml} / \mathrm{min} / 1.73 \mathrm{~m}^{2}\right)$

- $\quad$ Stage4; severe reduction in GFR (15$29 \mathrm{ml} / \mathrm{min} / 1.73 \mathrm{~m}^{2}$ )

- $\quad$ Stage 5; kidney failure with GFR $\left(<15 \mathrm{ml} / \mathrm{min} / 1.73 \mathrm{~m}^{2}\right)$

Nevertheless, the global increase in the incidence and prevalence of CKD is being driven by theglobal increase in the prevalence of diabetes mellitus, hypertension, obesity and aging. ${ }^{3}$ Globally CKD represents the $12^{\text {th }}$ cause of death and $17^{\text {th }}$ cause of disability. ${ }^{4.5}$ it is almost a truism to say that patients with end-stage renal failure (ESRD) are thought to represent the tip of the iceberg of the entire burden of CKD. ${ }^{1}$

The major common causes of the CKD in our environment include hypertension, diabetes mellitusand chronic glomerulonephritis.

Disorders of mineral metabolism, secondary hyperparathyroidism, hyperphosphatemia, hypocalcaemia and deficiencies of vitamin D are common complications of CKD. Elevations in the calcium-phosphorus product are clearly associated with calcification of coronary arteries and with cardiovascular-related mortality in patients undergoing dialysis. ${ }^{6}$ Cardiovascular-related deaths remain the primary cause of mortality in patients with end-stage renal disease. Hyperphosphataemia, hyper- or hypocalcaemia, hypomagnesaemia and increased parathyroid hormone (PTH) plasma concentrations, all commonly observed in CKD, are also associated with increased mortality. ${ }^{7}$ Thus, prevention of the disturbances in mineral and bone metabolism and their management early in the course of CKD are vital for improving the patient's quality of life and longevity.

\section{MATERIALSAND METHOD}

This study was conducted at the departments of Chemical Pathology and Medicine of Ahmadu Bello University Teaching Hospital, Shika - Zaria within fifteen months. One hundred and twentyfive (125) consecutive adult patients 18years and above in various stages of CKD who presented at the nephrology clinic of ABUTH Shika Zaria were enrolled into the study. Equal number of apparently healthy matched for sex and age controls were also recruited. Patient with acute renal failure, primary hyperparathyroidism and those who are pregnant were excluded from the study.

Approval was obtained from Ethical and Scientific committee of ABUTH Zaria before embarking on thestudy.

Fivemillilitres $(5 \mathrm{~mL})$ blood specimen was collected from a prominent peripheral vein. Theantecubital fossa was cleaned with $70 \%$ methylated spirit and allowed to dry. Blood was taken using a sterilized 5 $\mathrm{mL}$ syringe and $21 \mathrm{G}$ needle. $5 \mathrm{ml}$ of the blood sample was transferred into a plain sample bottle and allowed to stand for 30 minutes to dot and thereafter was centrifuged at 4000rpm for $10 \mathrm{~min}$. The serum was separated immediately and transferred into a serum bottle. Analysis of serum calcium was carried out immediately and the remaining serum was stored frozen at $-20^{\circ} \mathrm{C}$ until timefor analysis.

The data obtained were cleaned and entered into Statistical Package for the Social Sciences 17.0 (SPSS 17.0) for windows (SPSS Inc. Chicago, 17). Results were expressed as absolute numbers, percentages, means and standard deviations and ranges as appropriate. Serum calcium, phosphate, magnesium, vitamin $D$, parathyroid and creatinine obtained from the patients were compared with those of thecontrolsusing thetwo tailed Student'sttest. $p$-value of equal to or less than $0.05(p \leq 0.05)$ was considered statistically significant. Categorical data were presented as frequencies and percentages while continuous data were summarized as mean \pm standard deviation. Discrete variables were analysed using $X^{2}$. Students-t-test was used to analyse continues normally distributed variables. 
Thechemicalsand kitsused for themeasurement of serum calcium, phosphate, magnesium, creatinine, were procured from LABKIT Barcelona while that for parathyroid hormone and vitamin $D$ were procured fromWKEA medical supplies(China).

Adequate quality control was observed when carrying out the analysis of the samples to ensure that the results obtained are reliable. This was done by analysing the samples in batches together with quality control sera from labkit for specific analytes using multi control. Pooled sera were used for parathyroid hormoneand vitamin D.

\section{RESULTS}

One hundred and twenty-five (125) CKD patients aged 18to 85 years wereenrolled for thestudy CKD patients were in different stages, with $9 \%$ in stage 1 , $16 \%$ in stage $2,22 \%$ in stage $3,12 \%$ in stage 4 and $41 \%$ in end stage renal disease (stage 5) (table 1). Hypertensive nephropathy is the leading cause of CKD with $45.6 \%$, followed by diabetic nephropathy and adult polycystic kidney disease occurring with similar frequency of $16.8 \%$ each, while chronic glomerulonephritis accounted for the fourth cause with a frequency of $12 \%$. Other sundry causes comprising sickle cell nephropathy, etc accounted for $5.6 \%$.

Treatment modalities of CKD patients indicated that majority of the patients were on conservative treatment with108 (86.4\%) on conservative treatment, and $16(12.8 \%)$ were on dialysis and $1(0.8 \%)$ had transplant.

Serum ionised calcium, vitamin $\mathrm{D}$ and $\mathrm{eCrCl}$ showed a progressive decline as the stage of CKD advances, while serum levels of phosphate, creatinineand IPTH showed a progressiveincrease as the stage of CKD advances. Serum magnesium showed a slight changeeven with advancing stages of CKD. The difference in mean serum levels of calcium, phosphorus, vitamin D, parathyroid hormone, creatinine and estimated creatinine clearance with different stages of CKD were statistically significant ( $p<0.05$ ). (table 2 ) Serum ionised calcium showed statistical significance difference between stages 1 and 4, 1 and 5, 2 and 4, 2 and 5 and stage 3 and 5 . Serum phosphorus showed statistical significance difference between stages1 and 4, 1 and 5, 2 and 4, 2 and 5, 3 and 4, 3 and 5 and stage 4 and 5 . Serum vitamin $D$ and intact parathyroid hormone showed statistical significance difference between stages 1 and 3, 1 and 4,1 and 5,2 and 3,2 and 4,2 and 5 and stages 3 and 5 . The difference in estimated creatinine clearance and serum creatinine were statistically significantacrossall thestages of CKD. (Table3)

Table 4 showed that Estimated creatinine clearance $(\mathrm{eCrCl})$ correlated negatively with phosphate $(r=$ $0.350, p=0.00)$, and iPTH ( $r=-0.621, p 0.00$ ) while serum creatinine correlated negatively with calcium $(r=-0.599, p=0.00)$ and positively with phosphate $(r=+0.526, p=0.00)$ and iPTH $(r=$ $+0.449, p=0.00$ ).

\section{Table 1. Stages of CKD in studied patients}

\begin{tabular}{lll}
\hline STAGES & $\mathrm{n}$ & Percentage (\%) \\
\hline 1 & 11 & 9 \\
2 & 20 & 16 \\
3 & 27 & 22 \\
4 & 15 & 12 \\
5 & 52 & 41 \\
Total & 125 & 100 \\
\hline $\mathrm{n}=$ frequency & CKD =Chronic & $\%=$ percentage \\
& Kidney Disease
\end{tabular}


TABLE 2. Serum levels (mean \pm SD) of measured analytes in different stages of CKD

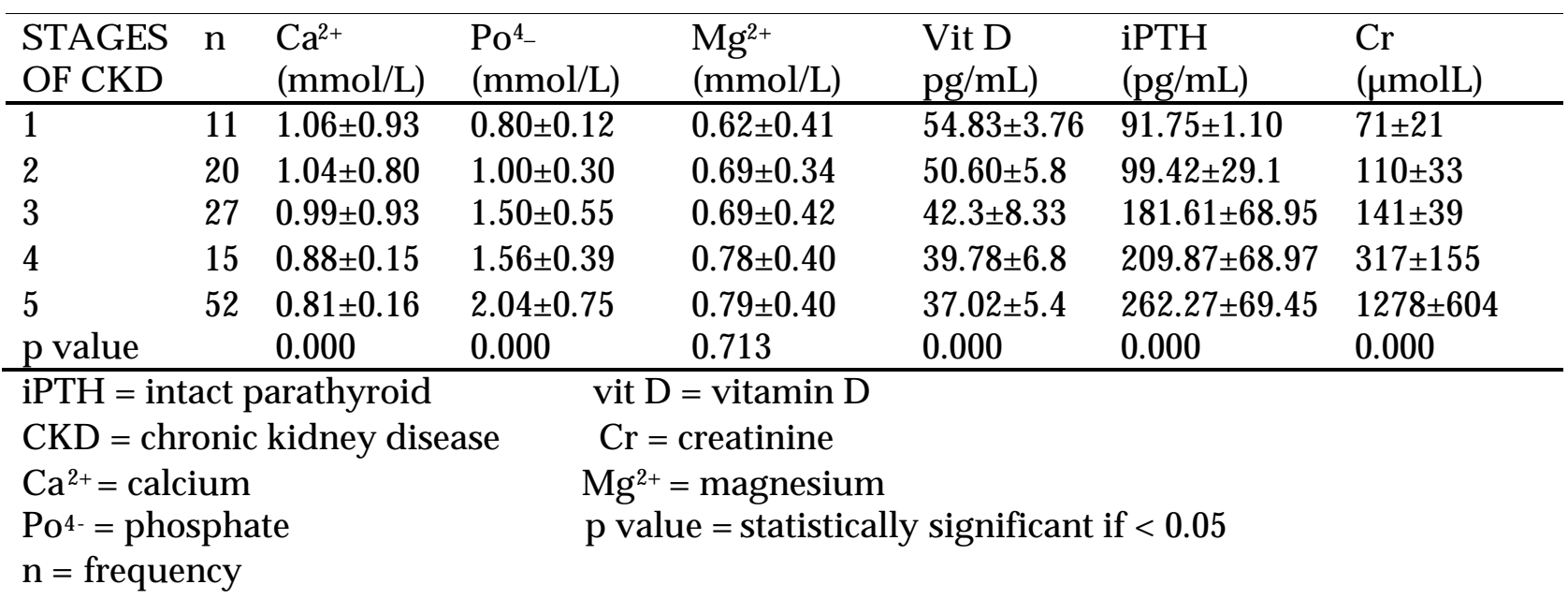

Table 3. Post hoc analysis between the studied analytes and various stages of CKD.

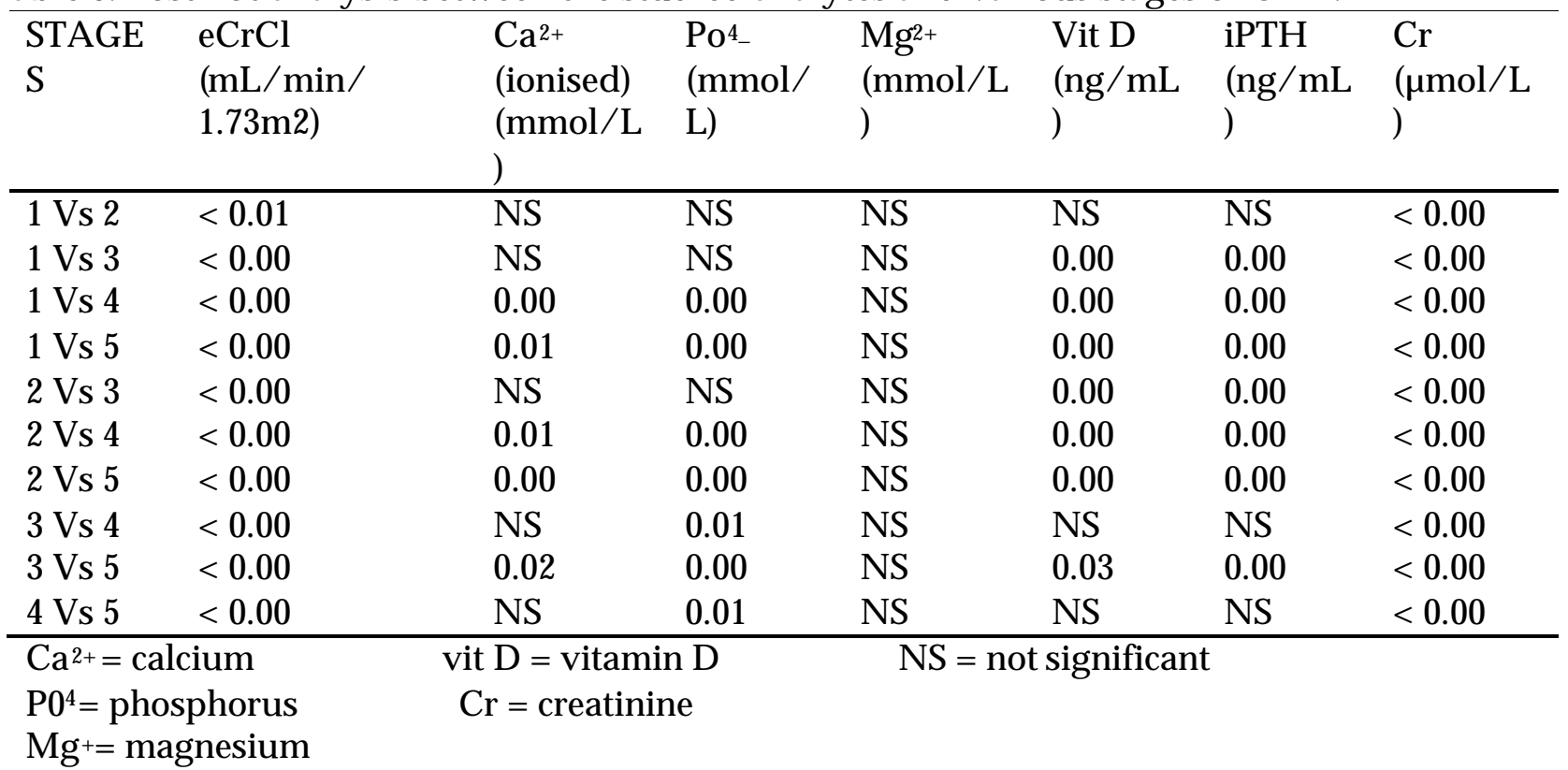

Table 4. Correlation between the different measured analytes in CKD patients

\begin{tabular}{|c|c|c|}
\hline V ariables & $r$ - value & $p$ - value \\
\hline $\mathrm{Po}^{4-} / \mathrm{eCrCl}$ & -0.340 & 0.00 \\
\hline iPTH/ eCrCl & -0.621 & 0.00 \\
\hline Po4-/ iPTH & 0.332 & 0.00 \\
\hline iPTH/ creatinine & 0.449 & 0.00 \\
\hline $\mathrm{Ca}^{2+} / \mathrm{iPTH}$ & -0.325 & 0.00 \\
\hline $\mathrm{Ca}_{2}+\mathrm{Po}_{4}$ & -0.410 & 0.00 \\
\hline $\mathrm{Ca}^{2+} /$ creatinine & -0.599 & 0.00 \\
\hline Po4-/ creatinine & 0.520 & 0.00 \\
\hline
\end{tabular}




\section{DISCUSSION}

Majority of the patients presented in late stages of CKD, with over $40 \%$ in ESRD requiring any form of kidney replacement treatment. Such trend was observed in thestudies conducted in South Western Nigeria by Akinsola et al, in Ile-lfe $2004^{8}$ and A lebiosu et al, in Sagamu 2006. ${ }^{9}$ who also indicated in their series that majority of patients were in advanced stages of CKD. This is hardly surprising since CKD is asymptomatic in the early stages coupled with poor health seeking behaviour in this part of thecountry.

The high mean serum creatinine and $\mathrm{eCrCl}$, found in patients with CKD in thisstudy wassimilar to the findings of Chijioke et al, in Ilorin 2011 who also estimated $\mathrm{eCrCl}$ by the Cockroft and Gault formula. ${ }^{10}$ This high mean creatinine levels may reflect the late presentation of majority of CKD patients in the study. This was similarly reported by Ifeoma etal, in Enugu in 2010 whileassessing the enormity of CKD in south eastern Nigeria. ${ }^{4}$ This might be because many of the patients with advanced CKD were managed conservatively because they could not afford the cost of renal replacement therapy (RRT). The mean serum magnesium levels in patients showed no significant difference between the various stages of CKD neither was there any correlation with other analytes. Thisis similar to the work of Ortega et al in 2013 in Spain who found all the CKD patients in stages 4 and 5 (study population) had normal baseline serum magnesium and there was no correlation with serum calcium, phosphorus and parathyroid hormone. ${ }^{11}$ The regulation and elimination of magnesium in patients with renal disease is somewhat understudied. However, in moderate CKD, the increase in the fractional excretion of magnesium compensates for the loss of renal function, such that serum levels are maintained within thenormal range.
Thefinding of thisstudy regarding $\mathrm{iPTH}$ and $\mathrm{eCrCl}$ was comparable with a previous study done by Rahman et. a,l in india, in 2005, who correlated the serum parathyroid hormonelevel with biochemical parameters in chronic renal failure in children. ${ }^{12}$ Ian $\mathrm{H}$. de Boer et al, also observed increased iPTH levels as the stage of CKD advances. ${ }^{13}$ This study has further confirmed that high PTH is a prominent feature in CKD as documented by Staloposky et al, (1996). ${ }^{14}$ The increased levels of mean serum iPTH may be related to reduction in serum calcium level and progressive rise of serum inorganic phosphate from early to advanced renal failure.

Looking at Renal capacity of handling calcium varies in the different stages of CKD, from at least CKD Stage 3 and onwards this trend was noticed in this work with increasing levels from stage 3 onwards. Other factors taken into consideration could be attributed to the black race and other factors other than the traditional markers like FGF23.The findings in this study on the correlation of biochemical analytes with stages of CKD with the exception of vitamin D agrees with that of Rahman et al, in 2005 and also with that of Malawadi et al, in India 2014. 12,15

Hypocalcaemia, hyperphosphataemia and hyperparathyroidism cuts across thestages of CKD with the exception of stage 1 while deficiency of vitamin $\mathrm{D}$ and magnesium derangement was noted in stages 3, 4 and 5. A greater percentage of hypocal caemia occurred in stage 5 no comparable local Nigeriastudy was found in literature search.

\section{CONCLUSION}

From this present study, it was found that majority of the CKD patients are in the late stage of CKD with correlation of analytes more evident in the late stages of the disease. Biochemical derangements occurred in late, rather than early stages of CKD.

2. Foundation, National Kidney. "KDOQI clinical practice guideline for diabetes and CKD: 2012 Update." American Journal of Kidney Diseases 60.5(2012): 850-886

3. Olugbenga EA, Alebiosu CO. Burden of chronic kidney disease; An international perspective. ACKD J 2010;17(3);215-224 
4. Ifeoma I U, Chinwuba K I, The enormity of chronic kidney disease in Nigeria: The situation in a teaching hospital in south east Nigeria. J Trop Med. 2010; 2010:1-6

5. World health organization," global burden of diseasemarch, 2006.

6. Block GA, Klassen PS, Lazarus JM, Ofsthun N, Lowrie EG, Chertow GM. Mineral metabolism, mortality and morbidity in maintenance haemodialysis. J Am Soc N ephrol 2004; 15(8):2208-18.

7. Block GA, Hulbert-Shearon TE, Levin NW, Port FK. Association of serum phosphorus and calcium $x$ phosphate product with mortality risk in chronic haemodialysis patients: a national study. Am J Kidney Dis 1998; 31(4):607-617.

8. Akinsola W, Odesanmi WO, Ogunniyi JO, Ladipo GO. Diseases causing chronic renal failure in Nigerians-a prospective study of 100 cases. Afr J Med Sci 1989; 18:131-7.

9. Alebiosu CO, Ayodele OO, Abbas A, Olutoyin A. Chronic renal failure at the Olabisi Onabanjo university teaching hospital, Sagamu, Nigeria. Afr Health Sci 2006;6(3):132-8.

10. Chijioke, A., A deribigbe, A., Olarenwaju, T. O., Makusidi, A. M., Oguntoyinbo, A. E. Prevalence and pattern of cystic kidney diseases in Ilorin, Nigeria. Saudi J Kidney Dis Transpl 2010 21(6), 1172.
11. Ortega, Olimpia, Isabel Rodriguez, Gabriela Cobo, Julie Hinostroza, PalomaGallar, Carmen Mon, Milagros Ortiz et al. "Lack of Influence of Serum Magnesium Levels on Overall Mortality and Cardiovascular Outcomes in Patients with Advanced Chronic Kidney Disease." IntScholarly Res N otices 2013(2013).

12. Rahman MH, Hosain MM, Sultana S, Jamal CY, Karim MA. Correlation of Serum parathormone level with biochemical parameters in chronic renal failure. Indian Pediatrics 2005; 42:250-54.

13. Ian $\mathrm{H}$, IninaGorodetskaya, Belinda Young, Chi-yuan, Glenn MC. The severity of secondary hyperparathyroidism in chronic renal insufficiency is GFR dependent, race dependent and associated with cardiovascular disease. J Am Soc Nephrol 2002; 13: 2762- 69.

14. Slatopolsky E, Firch J, Denda M, Ritter C, Zhong $M$, Dusso $A$, et al. Phosphate restriction prevents parathyroid gland growth. High phosphorus directly stimulates PTH secretion invitro. J Clin Invest 1996; 97(11):2534.

15. Malawadi, BN, SumaMN , PrashantV, A kila $P$, Anjalidevi BS, Manjunath S. "Secondary hyperparathyroidism in all the stages of chronic kidney disease in southern indian population."IJPPS2014; 4:6

Cite this article as: Mohammed A, Suleiman HM, Yusuf R, Aliyu IS, Ibrahim A, Jibril ME, Manu A. Relationship Between Serum Level of Ionized Calcium, Magnesium, Phosphate, Vitamin D and Parathyroid Hormone With Stages of CKD.

KJMS 2020; 14(1): 50 - 55. 